\title{
Quantifying the real life risk profile of inhaled corticosteroids in COPD by record linkage analysis
}

\author{
Rob W Flynn' ${ }^{1}$,Thomas M MacDonald ${ }^{1}$, Adrian Hapca', Isla S MacKenzie ${ }^{1}$ and Stuart Schembri2*
}

\begin{abstract}
Background: Inhaled corticosteroids (ICS), especially when prescribed in combination with long-acting $\beta_{2}$ agonists have been shown to improve COPD outcomes. Although there is consistent evidence linking ICS with adverse effects such as pneumonia, the complete risk profile is unclear with conflicting evidence on any association between ICS and the incidence or worsening of existing diabetes, cataracts and fractures. We investigated this using record linkage in a Dundee COPD population.

Methods: A record linkage study linking COPD and diabetes datasets with prescription, hospitalisation and mortality data via a unique Community Health Index (CHI) number. A Cox regression model was used to determine the association between ICS use and new diabetes or worsening of existing diabetes and hospitalisations for pneumonia, fractures or cataracts after adjusting for potential confounders. A time dependent analysis of exposure comparing time on versus off ICS was used to take into account patients changing their exposure status during follow-up and to prevent immortal time bias.

Results: 4305 subjects (3243 exposed to ICS, total of 17,229 person-years of exposure and 1062 non exposed, with a follow-up of 4,508 patient-years) were eligible for the study. There were 239 cases of new diabetes (DM) and 265 cases of worsening DM, 550 admissions for pneumonia, 288 hospitalisations for fracture and 505 cataract related admissions. The hazard ratio for the association between cumulative ICS and outcomes were 0.70 (0.43-1.12), 0.57 (0.24-1.37), 1.38 (1.09-1.74), 1.08 (0.73-1.59) and 1.42 (1.07-1.88) after multivariate analysis respectively.
\end{abstract}

Conclusion: The use of ICS in our cohort was not associated with new onset of diabetes, worsening of existing diabetes or fracture hospitalisation. There was however an association with increased cataracts and pneumonia hospitalisations.

Keywords: COPD, Inhaled steroids, Diabetes, Pneumonia, Cataract

\section{Background}

Chronic obstructive pulmonary disease (COPD) causes significant morbidity and mortality and is the third leading cause of death worldwide [1]. The aim of COPD treatment is to mainly reduce symptoms [2] as no intervention other than smoking cessation and supplemental oxygen has consistently been shown to improve mortality [3,4].

Inhaled corticosteroids (ICS), especially when prescribed in combination with long-acting $\beta_{2}$ agonists (LABA) improve quality of life (QoL), decrease exacerbations and hospitalisations, and have been associated with a trend towards a reduction in all-cause mortality [5]. However,

\footnotetext{
* Correspondence: sschembri@nhs.net

${ }^{2}$ Tayside Respiratory Research Group, East Block, Ninewells Hospital, Dundee DD1 9SY, UK

Full list of author information is available at the end of the article
}

unlike in asthma where the ICS dose is down titrated to the lowest possible that ensures symptom control, the ICS dose utilised in COPD can be quite high; a frequently used regime in the United Kingdom uses $500 \mu \mathrm{g}$ fluticasone propionate twice daily [6]. A recent Cochrane review confirmed that ICS therapy is associated with increased pneumonic events [7]. However the effect of ICS on other complications such as osteoporotic fractures, onset and progression of diabetes, glaucoma and cataracts is less clear [8-11].

In order to determine which patients should be prescribed ICS, the adverse event profile of ICS must be properly defined. Knowledge of potential risks is especially important in situations where a drug is frequently used outwith the specific group of patients that it should be 
targeted at. This is especially the case with ICS, as for example in Scotland, the Scottish Medicines Consortium has consistently advised that ICS should not be used for patients with COPD and a $\mathrm{FEV}_{1}>50 \%$ of predicted $[12,13]$, however, they are nevertheless widely used in patients outside these strict spirometric parameters [14].

We aimed to investigate the association between use of ICS in COPD and development of incident diabetes or worsening of prevalent diabetes, and of other adverse events.

\section{Methods}

This was a record-linkage cohort study using databases from Tayside Scotland. Data from the Tayside Medicines Monitoring Unit (MEMO) database is held within the Health Informatics Centre (HIC) [15]. This system collects data from the Tayside; a compact Scottish geographical area with a population of over 400,000 people. Health care for the region is co-ordinated by Tayside Health Board, which maintains a computerised record of all patients registered with a general practitioner (GP). In brief, the MEMO database contains several datasets including all dispensed community prescriptions, hospital discharge data, demographic data and biochemistry results. These data can be linked to disease-specific databases such as TARDIS (Tayside Allergy and Respiratory Disease Information System), DARTS (The Diabetes Audit and Research in Tayside Scotland; now called SCIDC) and other routine clinical data, all of which are linked by a Community Health Index (CHI) number that is unique to each patient.

\section{Data sources}

CHI master patient index - this defined the study population from which subjects were identified, providing data on registered GPs and dates that patients got registered, together with patients' date of birth and date of death.

$M E M O$ prescription dataset - this contains subject specific data on all prescriptions dispensed from community pharmacies in Tayside since 1993, including drug name, formulation, dosage, frequency and duration [15]. This provided data on the principal exposure of interest: ICS use. Other drug exposures used were other steroid use and anti-hypertensive medication.

Scottish Morbidity Records 1 (SMR01) - these data are routinely validated and collated by the Information and Services Division (ISD) of NHS Scotland and were available for Tayside from January 1, 1980 [15]. These contained diagnostic and procedural codes relating to all hospital inpatient episodes of care using the International Classification of Diseases ninth or tenth revisions (ICD-9, ICD-10) and Office of Population Censuses and Surveys Classification of Interventions and Procedures (OCSP4). These data were used to identify hospital admissions relating to pneumonia, fracture and cataract.

TARDIS - This has been described before [16]. GPs in Tayside refer patients with suspected COPD for screening spirometry. This is carried out in the GP practices by COPD nurses after structured training in order to obtain standardized results. COPD is diagnosed in patients with a $\mathrm{FEV}_{1}<80 \%$ of the predicted value (greatest of pre- and post-bronchodilator values) and $\mathrm{FEV}_{1} / \mathrm{FVC}<70 \%$. Patients with COPD are then invited to participate in TARDIS, a structured management programme. Patients are seen annually and relevant measures recorded then.

DARTS data - this is a validated population-based clinical information system of patients $(>8000)$ with diabetes in Tayside. Entries include attendances at hospital diabetes clinics, dispensed prescriptions for diabetes related medication and monitoring equipment, hospital discharge details, community-based mobile diabetic eye screening, glycosylated haemoglobin (HbA1c) and plasma glucose results from the regional biochemistry database. Validation against GP lists has confirmed DARTS to be robust [17].

\section{Study population}

Subjects resident in Tayside and registered with a GP between January 2001 and December 2012. They were censored if they died or left Tayside during the study period.

\section{Study subjects}

Subjects who registered with TARDIS database between January 2000 and December 2012 and who were 40 years old or over at diagnosis and who have at least two years of follow-up time formed the study cohort. The date of their first diagnosis of COPD (defined as having spirometry showing a $\mathrm{FEV}_{1} / \mathrm{FVC}<0.70$ ) was used as the study entry date. Patients who had a cancer diagnosis prior to the diagnosis of COPD were excluded from the study. Patients who developed cancer during the follow up time were censored one year prior to the diagnosis of cancer. For the primary analysis patients with type- 1 diabetes were excluded.

\section{Exposure}

Each dispensed ICS prescription has details of date of prescription, daily dose, amount and duration. ICS exposure was converted into beclometasone equivalent doses [6].

\section{Outcomes}

The primary outcome was either new cases of type 2 diabetes or worsening of pre-existing diabetes. These are defined below;

Newly diagnosed diabetes - new diagnosis of type 2 diabetes was recognised using the DARTS database, this has $95 \%$ sensitivity for identifying people with diabetes. 
Worsening of existing diabetic control - defined as either worsening of $\mathrm{HbA} 1 \mathrm{c}$ by $>5 \mathrm{mmol} / \mathrm{mol}$ or the prescription of additional hypoglycaemic agents, following the index visit.

Secondary endpoints - hospitalisations coded as pneumonia, fractures and cataracts were obtained from SMR1. The relevant ICD10 admissions and OPCS4 procedural codes are listed in Table $1[18,19]$.

\section{Covariates}

Covariates considered in the analysis consisted of other factors that could be potential confounders. These were: (1) Demographics (age, sex, social deprivation score, smoking status and BMI); (2) Severity of COPD: $\left(\mathrm{FEV}_{1}\right.$ and ratio of FEV1 /FVC at baseline, MRC dyspnoea score in the year before the first ICS use; (3) Disease history: cardiovascular risk defined as either primary or secondary prevention: primary prevention - hypertension (defined as on any anti-hypertension drug; treatment) and dyslipidaemia (defined as serum total cholesterol $>5 \mathrm{mmol} / \mathrm{L}$ ). Secondary prevention - myocardial infarction, heart failure, stroke, peripheral vascular disease (defined as hospitalisation from the SMR1 database). Renal disease was defined as having serum creatinine $\geq 220 \mu \mathrm{mol} / \mathrm{l}$. For the secondary outcomes, a prior history of the outcome "event" at baseline was included in the model.

\section{Statistical analysis}

Baseline characteristics were summarised as mean (SD)/ median (IQR) for continuous variables and number of subjects (\%) for categorical variables. These were compared using t-test for continuous variables and chi-square test for binary variables. A Cox regression model was used to explore the relationship between ICS exposure and the primary and secondary outcomes. Each endpoint was performed as a separate analysis allowing the calculation of relative risks associated with ICS use for each of the outcomes. A time dependent analysis of exposure - comparing time "on" versus "off" ICS exposure as judged by dispensed prescribing - was used to take into account the fact that patients changed their exposure status during follow-up and to prevent immortal time bias. The nature of the association between the exposure and outcome was

Table 1 Coding systems and specific codes used to identify study endpoints

\begin{tabular}{ll}
\hline Outcome & Coding system and codes used \\
\hline Pneumonia & ICD10: J12, J15, J16, J17, J18 \\
Fractures & ICD10: S02, S12, S22, S32, S42, S52, S62, S72, S82, S92 \\
Cataracts & OSCP4: C71, C72, C73, C74, C75 \\
& ICD 10 code:H25 \\
\hline
\end{tabular}

ICD10 - International Statistical Classification of Diseases 10th revision. OSCP4 - Office of Population Censuses and Surveys Classification of Surgical Operations 4th revision. incorporated into the time dependent model following that thought most biologically plausible: for cataract and osteoporosis this was cumulative steroid exposure; for pneumonia was current exposure; whilst for diabetes both cumulative and current exposure were modelled as there is no clear mechanism.

Both univariate and multivariate analyses were carried out. The multivariate analyses included all covariates thought to be potential confounders in an attempt to establish the true treatment effect. Variables included in the statistical models were potential predictors of the outcome events. Other covariates were evaluated once at the start of the follow up period. For the pneumonia outcome the population attributable risk was calculated by multiplying (hazard ratio-1)/hazard ratio by the probability of disease given exposure.

The exposed cohort was followed up from the date of first exposure to ICS. Non-ICS exposed patients were followed up from a randomly generated surrogate date of first "exposure" generated using a frequency-matched calendar year date of exposure. All statistical analyses were carried out using SAS (version 9.2).

There were very few missing data in the record linked data: $(1.1 \%$ deprivation score, $4.4 \%$ BMI, 4.6\% dyspnoea score). Where covariates did have missing values a complete case analysis performed. To establish if there were any relevant interactions in the multivariate analyses, two-way interactions terms were modelled incorporating ICS exposure and other key covariates of interest.

\section{Sensitivity analyses}

A number of sensitivity analyses were carried out to test the robustness of the finding. First we used an inception cohort - consisting of only those patients naive to ICS at the start of follow-up.

Propensity score matching was used to explore possible confounding by indication that was not adequately controlled for by the Cox model. In view of data showing that the effect of ICS on pneumonia is no longer evident 6 months after drug cessation [20], analyses were also run using an extended screening period of 6-months to test the stability of the models generated and to see if there was any evidence of a carryover effect. The primary analysis considered any dose of ICS, versus none. As sensitivity analysis we also attempted to establish if there was a dose-response between exposure and outcome at zero, low, medium and high ICS doses. To standardise different doses of ICS we mapped potency as budesonide = beclametasone $=50 \%$ fluticasone (i.e. $1 \mathrm{mcg}$ budesonide $=1 \mathrm{mcg}$ beclometasone $=0.5 \mathrm{mcg}$ fluticasone) [6]. This approach allowed the dose response of the different ICS products to be compared. Similarly this standardisation was also used to allow comparison of the different steroids, to see if it 
was possible to detect a difference between outcome and the type of steroid used (i.e. fluticasone vs "other" beclometasone, budesonide etc).

To assess the impact of "other" (non-inhaled) steroid use, a sensitivity analysis was also conducted which stratified the cohort by extent of other steroid use. Finally we also repeated analyses using a fully time dependent analysis starting from point of diagnosis on TARDIS for all patients rather than the date of first exposure (or "surrogate date" of exposure as described).

\section{Results}

We identified 4,305 subjects as being eligible for the study cohort. Figure 1 details the number of patient available on the TARDIS database and details on excluded patients. 3,243 were exposed to ICS for a total of 17,229 personyears of exposure, and 1,062 were unexposed with a follow-up of 4,508 years. A comparison of the baseline characteristics of the exposed and unexposed cohorts is shown in Table 2. Fluticasone was responsible for $67.7 \%$ of the ICS prescription exposure and beclometasone for $20.5 \%$. The remaining ICS prescriptions (11.8\%) were for budesonide. There were 239 cases of newly diagnosed and 265 cases of worsening of existing diabetes. For the secondary outcomes there were 550 admissions for pneumonia, 288 hospitalisations for fracture and 505 cataract related admissions. The number of events by exposure group and exposure time is shown in Table 3. This shows the nature of the risk for the two components of the primary endpoint to be quite different, with the new onset diabetes being uncommon across a large number of patients, whilst the worsening of diabetes outcome was extremely common across a small number of patients. For this reason these two endpoints were treated separately for the remaining analyses.

The results of the multivariate analyses are shown in Table 4. These show that there was no association between ICS use and either onset of new cases of diabetes or cases of worsening of existing diabetes. This was consistent across the whole range of sensitivity analyses that were run. For the secondary endpoints a consistent association was found between ICS and increased risk of both hospital admissions for pneumonia and cataract, but not fracture. The sensitivity analyses using models that incorporated various drug exposure screening periods found evidence of a carryover effect for the pneumonia outcome. This implies that the association between ICS use and hospitalisation for pneumonia continues to be present for some time after the ICS use has ceased (Tables 4 and 5). The population attributable risk associated with the use of ICS is an extra 7.4 pneumonia hospitalisation per 1,000 person years of exposure, with the results that of the 550

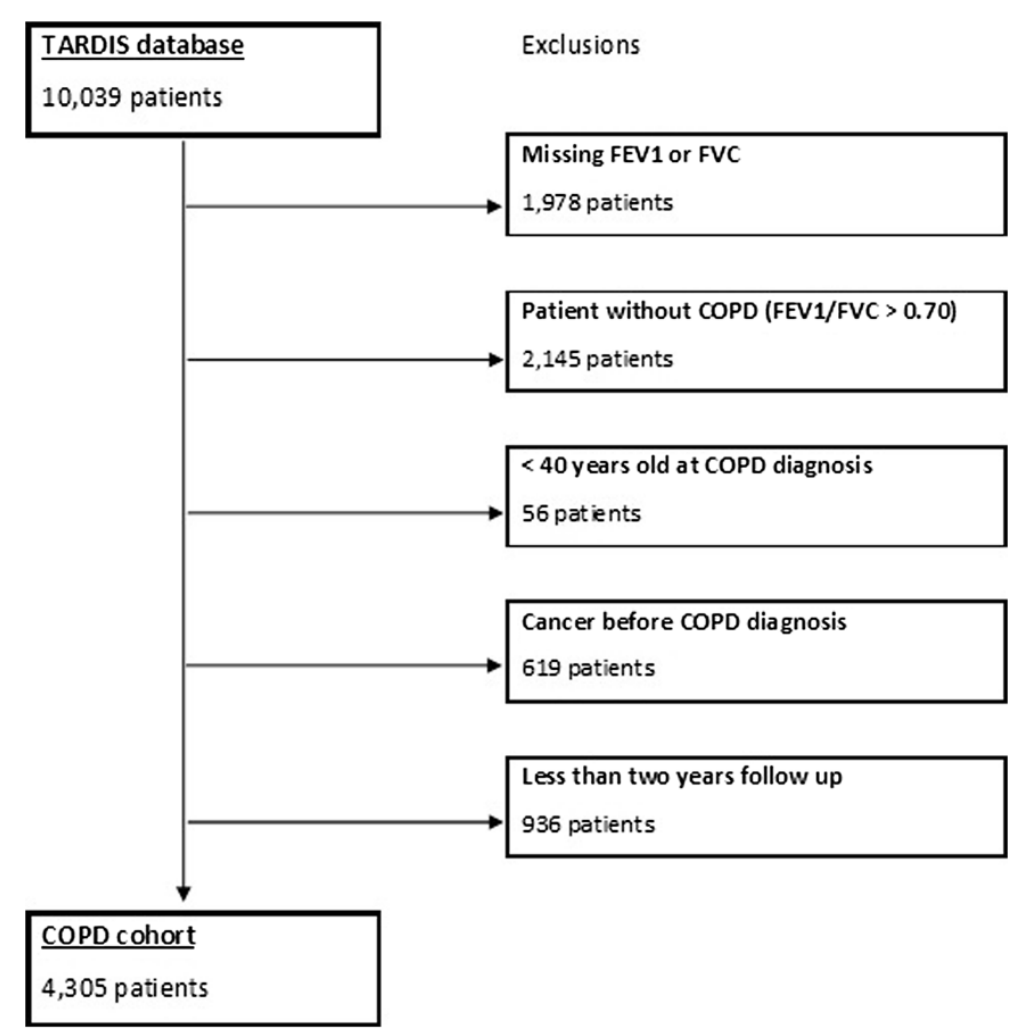

Figure 1 CONSORT diagram showing derivation of study cohort. 
Table 2 Comparison of the baseline characteristics of the ICS exposed and unexposed cohorts

\begin{tabular}{|c|c|c|c|}
\hline Continuous variables & $\begin{array}{l}\text { ICS exposed } \\
(n=3243)\end{array}$ & $\begin{array}{l}\text { ICS un-exposed } \\
(n=1062)\end{array}$ & p value ${ }^{\#}$ \\
\hline Mean age (years) & 65.5 & 67.2 & $<0.0001$ \\
\hline Mean BMI (kg/m2) & 26.3 & 26.6 & 0.1772 \\
\hline Smoking (pack years) & 34.7 & 39.0 & $<0.0001$ \\
\hline Social deprivation (Scottish index of multiple deprivation; $1=$ most deprived, $5=$ most affluent) & 2.31 & 2.29 & 0.5972 \\
\hline Dyspnoea score ( 1 = least breathless, 5 = most breathless $)$ & 2.57 & 2.19 & $<0.0001$ \\
\hline Gender (female) & $51.9 \%$ & $49.5 \%$ & 0.1693 \\
\hline Primary CV prevention (hypertension, dyslipidaemia) & $73.4 \%$ & $79.0 \%$ & 0.0003 \\
\hline Secondary CV prevention (myocardial infarction, heart failure, stroke, PVD) & $11.3 \%$ & $15.6 \%$ & 0.0002 \\
\hline Renal diseases history & $2.9 \%$ & $2.5 \%$ & 0.4692 \\
\hline Diabetes history & $8.0 \%$ & $9.8 \%$ & 0.0711 \\
\hline Pneumonia history & $5.4 \%$ & $3.8 \%$ & 0.0375 \\
\hline Fracture history & $5.7 \%$ & $5.8 \%$ & 0.8413 \\
\hline Cataract history & $10.3 \%$ & $10.9 \%$ & 0.5840 \\
\hline Oral steroids prescription history & $58.9 \%$ & $30.0 \%$ & $<0.0001$ \\
\hline Rectal steroids prescription history & $11.8 \%$ & $10.4 \%$ & 0.1971 \\
\hline Topical steroids prescription history & $61.1 \%$ & $59.8 \%$ & 0.4540 \\
\hline $\mathrm{FEV}_{1}$ baseline (Litres) & $1.63(0.65)$ & $1.82(0.63)$ & $<0.0001$ \\
\hline Percent predicted FEV & $74.9(23.3)$ & $85.0(20.8)$ & $<0.0001$ \\
\hline $\mathrm{FEV}_{1} / \mathrm{FVC}$ ratio & $55.1(10.9)$ & $59.8(8.6)$ & $<0.0001$ \\
\hline
\end{tabular}

* "Percentage predicted” is baseline $\mathrm{FEV}_{1}$ expressed as percentage of predicted $\mathrm{FEV}_{1}$.

\#t-test for continuous variables and chi-square test for binary variables.

observed pneumonia hospitalisation in our study cohort 131 (23.8\%) could be attributed to use of ICS. A statistically significant interaction between age and sex was found for the pneumonia outcome only; other interaction terms were not significant. Table 5 shows the association between fluticasone alone and other ICS with outcomes. This shows that there were no differences between steroid groups and incidence of diabetes or fractures however there are increased pneumonic and cataract hospitalisations in the patients treated with fluticasone. Analysing only the group of patients on ICS showed that fluticasone had a significantly increased risk of pneumonia hospitalisation (HRs no carryover 1.39 (1.06-1.82), 180 day carryover 1.43 (1.14-1.78)) when compared with individuals on other ICS.

The extent of steroid use via all routes is shown in Table 6. This shows that there was widespread use of steroids administered by other routes. Indeed the majority of patient received greater exposure to oral than inhaled steroid. Use of rectal steroids was not common and the true extent to which systemic absorption of topical steroids is an issue is hard to assess. However the addition of oral steroid exposure to the models had little impact on the point estimates of the hazard ratios for the primary

Table 3 Number of events and year of follow-up for each of the study endpoints

\begin{tabular}{|c|c|c|c|c|c|c|}
\hline \multirow[t]{2}{*}{ Event } & \multicolumn{3}{|c|}{ ICS exposed $(n=3243)$} & \multicolumn{3}{|c|}{ ICS unexposed $(n=1062)$} \\
\hline & $n$ events & $\begin{array}{l}\text { Person-years } \\
\text { follow-up* }\end{array}$ & $\begin{array}{l}\text { Event rate } \\
\text { (per } 1000 \text { person years) }\end{array}$ & $n$ event & $\begin{array}{l}\text { Person-years } \\
\text { follow-up }\end{array}$ & $\begin{array}{l}\text { Event rate } \\
\text { (per } 1000 \text { person years) }\end{array}$ \\
\hline $\begin{array}{l}\text { New onset diabetes } \\
(n \text { at risk }=3,941)\end{array}$ & 186 & 16,634 & 11.2 & 53 & 4,285 & 12.4 \\
\hline $\begin{array}{l}\text { Worsening of existing diabetes } \\
\text { ( } n \text { at risk }=364 \text { ) }\end{array}$ & 194 & 595 & 326.1 & 71 & 223 & 318.4 \\
\hline Hospitalisation for pneumonia & 476 & 17,795 & 26.8 & 74 & 4,833 & 15.3 \\
\hline Hospitalisation for fracture & 234 & 18,097 & 12.9 & 54 & 4,822 & 11.2 \\
\hline Cataract & 411 & 17,314 & 23.7 & 94 & 4,581 & 20.5 \\
\hline
\end{tabular}

*"person years follow-up" is the number of years exposure / non-exposure during which subjects were at risk of suffering the event of interest. This varies depending on the number of subjects in the cohort and the number / timing of any events that occurred. 
Table 4 Association between inhaled steroids and outcomes

\begin{tabular}{llll}
\hline Outcome & $\begin{array}{l}\text { Univariate } \\
\text { hazard ratio } \\
(95 \% \mathrm{Cl})\end{array}$ & $\begin{array}{l}\text { Multivariate } \\
\text { hazard ratio } \\
(95 \% \mathrm{Cl})\end{array}$ \\
\hline
\end{tabular}

\section{Primary endpoints}

New onset diabetes

$\begin{array}{lll}\text { Any exposure } & 0.88(0.68-1.14) & 0.94(0.71-1.25) \\ \text { Cumulative exposure } & 0.71(0.46-1.10) & 0.70(0.43-1.12)\end{array}$

\section{Worsening of existing diabetes}

Current exposure $\quad 0.94(0.73-1.21) \quad 0.91(0.73-1.21)$

Cumulative exposure $\quad 0.57(0.25-1.30) \quad 0.57(0.24-1.37)$

\section{Secondary endpoints}

\section{Hospitalisation for pneumonia}

Current exposure no $\quad 1.27(1.07-1.50) \quad 1.13(0.94-1.36)$ carryover

Current exposure with $1.58(1.29-1.93) \quad 1.38(1.09-1.74)$ 180-day carryover

\section{Hospitalisation for fracture}

Cumulative exposure $\quad 1.06(0.75-1.51) \quad 1.08(0.75-1.51)$

\section{Cataract related outcome}

Cumulative exposure $\quad 1.43(1.11-1.83) \quad 1.42(1.07-1.88)$ history*, secondary CV history, renal dysfunction, COPD severity*, history of diabetes*, history of cataract related admission

Age $^{*}$, sex, deprivation*, previous ICS, history of oral steroid, smoking*, BMI*, primary CV history*, secondary CV history*, renal dysfunction, COPD severity*, history of diabetes*, history of pneumonia admission*

Age $^{*}$, sex* ${ }^{*}$ deprivation, previous ICS, history of oral steroid, smoking, BMI*, primary CV history, secondary CV history, renal dysfunction, COPD severity, history of diabetes, history of fracture admission*

Age $^{*}$, sex, deprivation, history ICS, history oral steroids*, smoking, BMI*, primary CV history*, secondary CV history, renal dysfunction, COPD severity

Age $^{*}$, sex, deprivation, previous ICS, history of oral steroid use, smoking, BMI, primary CV history, secondary CV history, renal dysfunction, COPD severity*

Age*, sex, deprivation, previous ICS, history of oral steroid, smoking, BMI, primary CV history, secondary CV history, renal dysfunction, COPD severity*

*Shows variables that were found to be significant in the model.

Abbreviations: $\mathrm{Cl}$ confidence interval, ICS inhaled corticosteroids, BMI body mass index, CV cardiovascular, COPD chronic obstructive pulmonary disease.

"COPD severity" is the actual FEV 1 and a percentage of predicted FEV 1

and secondary endpoints. The other sensitivity analyses yielded results that had no impact of the main finding of this study. The inception cohort analyses resulted in a sufficiently small cohort that results tended to be nonsignificant. The propensity score analyses showed nothing to suggest that they offered any additional adjustment for confounding. Similarly the other sensitivity analyses undertaken showed the study findings to be robust.

\section{Discussion}

Our results show that the use of ICS in our cohort was not associated with new onset of diabetes or worsening

Table 5 Comparison of effect of fluticasone and "other ICS" on outcomes

\begin{tabular}{|c|c|c|c|}
\hline \multirow[t]{2}{*}{ Outcome } & \multicolumn{3}{|c|}{$\begin{array}{l}\text { Multivariate hazard ratio } \\
(95 \% \mathrm{Cl})\end{array}$} \\
\hline & No ICS & Other ICS & Fluticasone \\
\hline \multicolumn{4}{|l|}{ Primary endpoints } \\
\hline New case diabetes & 1.00 & $0.98(0.69-1.39)$ & $0.82(0.58-1.15)$ \\
\hline Worsening of existing diabetes & 1.00 & $1.01(0.72-1.41)$ & $0.84(0.60-1.17)$ \\
\hline \multicolumn{4}{|l|}{ Secondary endpoints } \\
\hline \multicolumn{4}{|l|}{ Hospitalisation for pneumonia } \\
\hline No carryover & 1.00 & $0.88(0.68-1.14)$ & $1.23(1.01-1.51)$ \\
\hline 180-day carryover & 1.00 & $1.05(0.79-1.39)$ & $1.50(1.17-1.91)$ \\
\hline Hospitalisation for fracture & 1.00 & $1.08(0.72-1.60)$ & $1.10(0.57-2.10)$ \\
\hline Cataract related outcome & 1.00 & $1.34(0.99-1.82)$ & $2.24(1.40-3.60)$ \\
\hline
\end{tabular}


Table 6 Mean and median cumulative doses of all steroid routes for all patients

\begin{tabular}{llllll}
\hline Steroid exposure route & $\mathbf{n}$ & Mean & Median & $\begin{array}{l}\text { Inter quartile } \\
\text { range }\end{array}$ \\
\hline Cumulative inhaled dose & 3,243 & $0.45 \mathrm{~g}$ & $0.27 \mathrm{~g}$ & $0.01-0.69 \mathrm{~g}$ \\
Cumulative oral dose & 2,465 & $1.47 \mathrm{~g}$ & $0.25 \mathrm{~g}$ & $0-1.4 \mathrm{~g}$ \\
$\begin{array}{l}\text { Cumulative rectal dose } \\
\text { Cumulative number of }\end{array}$ & 257 & $0.01 \mathrm{~g}$ & $0 \mathrm{~g}$ & $0-0 \mathrm{~g}$ \\
$\begin{array}{l}\text { prescription for topical steroids } \\
\text { R }\end{array}$ & 1,966 & 24 & 0 & $0-32$ \\
\hline
\end{tabular}

of existing diabetes. Although systemic corticosteroids have a well recognised role in increasing diabetes risk [21], the impact of ICS is less well characterised and our study adds to the body of evidence in this field. Initial studies did not show an association between ICS use and diabetes [22,23], however a large population based study of 388,584 patients with respiratory disease (not just COPD) showed that ICS treatment was associated with a $34 \%$ increased risk of new onset diabetes (defined as initiation of an oral hypoglycaemic agent), with those on the highest ICS dose having the greatest risk [11]. More recently, a retrospective study of administrative claims data from the Australian Government Department of Veterans' Affairs of more than 18,000 patients with diabetes, found there to be an increased risk of diabetesrelated hospitalisations with the use of high-dose ICS [24]. Following this observational data, O'Byrne and colleagues examined double-blind, placebo-controlled, trials in patients $\geq 4$ years of age with asthma or COPD involving budesonide or fluticasone to a lesser extent and did not find an association between inhaled corticosteroid treatment and increased risk of new onset diabetes mellitus or hyperglycaemia [25]. This is consistent with our findings and suggests that any effect of ICS on diabetes in COPD patients, if present at all, is unlikely to be clinically significant.

The relationship between ICS and pneumonia risk was first noted in TORCH with participants receiving ICS treatment having a two-fold higher rate of pneumonia when compared with those in the placebo arm [5]. These events were often diagnosed and managed in primary care, our results show that ICS use was also associated with hospitalisation for pneumonia. It has been suggested that ICS type may influence the risk of pneumonia, with a recent Cochrane review and studies such as PATHOS suggesting that fluticasone carries a higher risk than others such as budesonide; this difference is also seen in our study $[7,26]$. Fluticasone's increased immunosuppressant potency (10-fold higher than that of budesonide with regard to ex vivo inhibition of human alveolar macrophage innate immune response to bacterial triggers) [27] could potentially explain these findings. As with other datasets we have shown that ICS use is associated with cataract surgery but not hospitalisation for fractures suggesting that there is no clinically significant association with osteoporosis $[9,28]$. Indeed a recent Cochrane review reported that ICS were not associated with an effect on fractures and bone mineral density [29] A previously unreported association was that fluticasone was more strongly associated with cataract surgery than other ICS, it is unclear why this may be the case however dose effect may be one explanation.

In order to justify prescribing ICS one must have data on the risk/benefit balance hence understanding the adverse effects of ICS is key. Although ICS have been shown to improve QoL, decrease exacerbations and hospitalisations and possibly improve mortality, evidence of benefit is limited to individuals with $\mathrm{FEV}_{1}<70 \%$ predicted [30]. Studies that solely recruited patients with milder disease did not show similarly improved outcomes [31] however several studies have shown that over half of ICS use in daily practice falls outwith strict spirometric parameters included in COPD guidelines [32].

The strengths of this study are that data were collected in routine care with minimal exclusions: this increases the likelihood that the results will be applicable to other populations. Another strength is that all COPD diagnoses were validated using spirometric data, to our knowledge this is the first observational study on the effects of ICS and diabetes in COPD that has done this. Despite data being collected prospectively according to predefined criteria, follow-up data are observational and therefore prone to the weaknesses of this type of study. Though we attempted to limit bias by adjusting for other potential confounders, bias due to unrecorded factors may remain.

\section{Conclusions}

ICS exposure in our cohort was not associated with new onset of diabetes, worsening of existing diabetes or fracture hospitalisation. There was however an association with increased cataracts and pneumonia hospitalisations. Knowing the adverse effect profile of ICS is especially important as they are often used in patients with no proven evidence of benefit.

\begin{abstract}
Abbreviations
ICS: Inhaled corticosteroids; COPD: Chronic obstructive pulmonary disease; CHI: Community health index; DM: Diabetes mellitus; LABA: Long-acting beta agonist; QoL: Quality of life; FEV1: Forced expiratory volume in one second; MEMO: Tayside medicines monitoring unit; HIC: Health informatics centre; GP: General practitioner; TARDIS: Tayside allergy and respiratory disease information system; DARTS: Diabetes audit and research in tayside; SMR01: Scottish morbidity records 1; ISD: Information and services division; ICD: the International classification of diseases; OCSP4: Office of population censuses and surveys classification of interventions and procedures (OCSP4); $\mathrm{HbA1c}$ : Glycosylated haemoglobin.
\end{abstract}

\section{Competing interests}

This study was funded by Novartis. TMM department holds research grants from Novartis, Pfizer, Amgen, Ipsen, Teijin \& Menarini, he is or has been the principal investigator on trials paid for by: Pfizer, Novartis, Ipsen, Teijin \& 
Menarini. Over the last 5 years TMM has been paid consulting or speakers fees by Pfizer, Novartis, Kaiser Permanante, Takeda, Servier, Shire, Astellus, Menarini, Daiichi, Sankyo \& AstraZeneca. Over the last 5 years SS has received funding from GlaxoSmithKline, Chiesi, Napp and Boehringer Ingelheim to attend meetings. He has been a consultant to Chiesi, Novartis and Bayer. SS is or has been the principal investigator on trials paid for by: GlaxoSmithKline, Novartis and Nycomed. ISM is or has been the principal investigator on trials paid for by: Novartis. RWVF and $\mathrm{AH}$ declare no competing interests.

\section{Authors' contributions}

All authors were involved in design of the study, analysis and interpretation of results, and preparation and revision of the manuscript. All authors read and approved the final manuscript.

\section{Acknowledgements}

Dorothy Keininger, Pablo Altman, and David Young provided comments on a draft report and the manuscript however did not meet the ICMJE criteria for authorship (www.icmje.org/).

\section{Author details}

${ }^{1}$ Medicines Monitoring Unit, Ninewells Hospital and University of Dundee, Dundee DD1 9SY, UK. ²Tayside Respiratory Research Group, East Block, Ninewells Hospital, Dundee DD1 9SY, UK

Received: 10 July 2014 Accepted: 29 October 2014

Published online: 19 November 2014

\section{References}

1. Lozano R, Naghavi M, Foreman K, Lim S, Shibuya K, Aboyans V, Abraham J, Adair T, Aggarwal R, Ahn SY, Alvarado M, Anderson HR, Anderson LM, Andrews KG, Atkinson C, Baddour LM, Barker-Collo S, Bartels DH, Bell ML, Benjamin EJ, Bennett D, Bhalla K, Bikbov B, Bin Abdulhak A, Birbeck G, Blyth F, Bolliger I, Boufous S, Bucello C, Burch M, et al: Global and regional mortality from 235 causes of death for 20 age groups in 1990 and 2010: a systematic analysis for the Global

Burden of Disease Study 2010. Lancet 2012, 380:2095-2128.

2. National Collaborating Centre for Chronic Conditions: Chronic Obstructive Pulmonary Disease: National clinical guideline on management of adults with chronic obstructive pulmonary disease in primary and secondary care. NICE guideline 12. Thorax 2004, 59(I):1-232.

3. Godtfredsen NS, Lam TH, Hansel TT, Leon ME, Gray N, Dresler C, Burns DM,

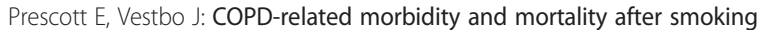
cessation: status of the evidence. ERJ 2008, 32:844-853.

4. Medical Research Council Working Party: Long-term domiciliary oxygen therapy in chronic hypoxic cor pulmonale complicating chronic bronchitis and emphysema. Lancet 1981, 1:681-686.

5. Calverley PM, Anderson JA, Celli B, Ferguson GT, Jenkins C, Jones PW, Yates JC, Vestbo J, TORCH investigators: Salmeterol and fluticasone propionate and survival in chronic obstructive pulmonary disease. N Engl J Med 2007, 356:775-789.

6. Joint Formulary Committee: British National Formulary. 67th edition. London: BMJ Group and Pharmaceutical Press; 2014.

7. Kew KM, Seniukovich A: Inhaled steroids and risk of pneumonia for chronic obstructive pulmonary disease. Cochrane Database Syst Rev 2014, 3. CD010115.

8. Garbe E, Suissa S, LeLorier J: Association of inhaled corticosteroid use with cataract extraction in elderly patients. JAMA 1998, 280:539-543.

9. Lipworth BJ: Systemic adverse effects of inhaled corticosteroid therapy: a systematic review and meta-analysis. Arch Intern Med 1999, 159:941-955.

10. Kelly HW, Nelson HS: Potential adverse effects of the inhaled corticosteroids. J Allergy Clin Immunol 2003, 112:469-478

11. Suissa S, Kezouh A, Ernst P: Inhaled corticosteroids and the Risks of Diabetes Onset and Progression. Am J Med 2010, 123:1001-1006.

12. Scottish Medicines Consortium. SMC No. (953/14; 2014 [ONLINE] Available at: www.scottishmedicines.org.uk/SMC_Advice/Advice/953_14_fluticasone_ furoate_vilanterol_Relvar/fluticasone_furoate_vilanterol_Relvar_Ellipta. [Accessed 03 November 14].

13. Scottish medicine consortium. SMC No. (4 50/08); 2008 [ONLINE] Available at: www.scottishmedicines.org.uk/files/salmeterol_fluticasone_inhaler_
Seretide_500_Accuhaler_FINAL_Feb_2008_for_website_amended_ 13_03_08.pdf. [Accessed 03 November 14]

14. Short PM, Lipworth SI, Elder DH, Schembri S, Lipworth BJ: Effect of $\beta$ blockers in treatment of chronic obstructive pulmonary disease: a retrospective cohort study. BMJ 2011, 342:d2549.

15. Wei L, Parkinson J, MacDonald TM: The Tayside Medicines Monitoring unit (MEMO). In Pharmacoepidemiology. 4th edition. Edited by Strom BL. Chichester: John Wiley and Sons; 2005:323-336.

16. Sheng X, Murphy MJ, MacDonald TM, Schembri S, Simpson W, Winter J, Winter $\mathrm{JH}$, Wei L: Effect of statins on total cholesterol concentrations, cardiovascular morbidity, and all-cause mortality in chronic obstructive pulmonary disease: a population-based cohort study. Clin Ther 2012, 34:374-384.

17. Morris AD, Boyle DI, MacAlpine R, Emslie-Smith A, Jung RT, Newton RW, MacDonald TM: The diabetes audit and research in Tayside Scotland (DARTS) study: electronic record linkage to create a diabetes register. DARTS/MEMO Collaboration. Br Med J 1997, 315:524-528.

18. World Health Organization: International classification of diseases, related health problems. 10th edition. Geneva: World Health Organization; 1992.

19. OPCS4 - Office of Population Censuses and Surveys: Tabular List of the Classification of Surgical Operations and Procedures Fourth Revision. London: Her Majesty's Stationery Office; 1990.

20. Suissa S, Patenaude V, Lapi F, Ernst P: Inhaled corticosteroids in COPD and the risk of serious pneumonia. Thorax 2013, 68:1029-1036.

21. McMahon M, Gerich J, Rizza R: Effects of glucocorticoids on carbohydrate metabolism. Diabetes Metab Rev 1988, 4:17-30.

22. Dendukuri N, Blais L, LeLorier J: Inhaled corticosteroids and the risk of diabetes among the elderly. Br J Clin Pharmacol 2002, 54:59-64.

23. Blackburn D, Hux J, Mamdani M: Quantification of the risk of corticosteroid-induced diabetes mellitus among the elderly. J Gen Intern Med 2002, 17:717-720

24. Caughey GE, Preiss AK, Vitry Al, Gilbert AL, Roughead EE: Comorbid diabetes and COPD: impact of corticosteroid use on diabetes complications. Diabetes Care 2013, 36:3009-3014.

25. O'Byrne PM, Rennard S, Gerstein H, Radner F, Peterson S, Lindberg B, Carlsson LG, Sin DD: Risk of new onset diabetes mellitus in patients with asthma or COPD taking inhaled corticosteroids. Respir Med. 2012 , 106:1487-1493.

26. Janson C, Larsson K, Lisspers KH, Ställberg B, Stratelis G, Goike H, Jörgensen $L$, Johansson G: Pneumonia and pneumonia related mortality in patients with COPD treated with fixed combinations of inhaled corticosteroid and long acting $\beta 2$ agonist: observational matched cohort study (PATHOS). BMJ 2013, 346:f3306.

27. Ek A, Larsson K, Siljerud S, Palmberg L: Fluticasone and budesonide inhibit cytokine release in human lung epithelial cells and alveolar macrophages. Allergy 1999, 54:691-699.

28. Ferguson GT, Calverley PM, Anderson JA, Jenkins CR, Jones PW, Willits LR, Yates JC, Vestbo J, Celli B: Prevalence and progression of osteoporosis in patients with COPD: results from the TOwards a Revolution in COPD Health study. Chest 2009, 136:1456-1465.

29. Yang IA, Clarke MS, Sim EH, Fong KM: Inhaled corticosteroids for stable chronic obstructive pulmonary disease. Cochrane Database Syst Rev 2012, 11:7. CD002991.

30. Dransfield MT, Bourbeau J, Jones PW, Hanania NA, Mahler DA, Vestbo J, Wachtel A, Martinez FJ, Barnhart F, Sanford L, Lettis S, Crim C, Calverley PM: Once-daily inhaled fluticasone furoate and vilanterol versus vilanterol only for prevention of exacerbations of COPD: two replicate doubleblind, parallel-group, randomised controlled trials. Lancet Respir Med 2013, 1:210-223.

31. Vestbo J, Sørensen T, Lange P, Brix A, Torre P, Viskum K: Long-term effect of inhaled budesonide in mild and moderate chronic obstructive pulmonary disease: a randomised controlled trial. Lancet 1999, 353:1819-1823.

32. Jebrak $G$, Initiatives B: COPD routine management in France: are guidelines used in clinical practice? Rev Mal Respir 2010, 27:11-18.

doi:10.1186/s12931-014-0141-y

Cite this article as: Flynn et al:: Quantifying the real life risk profile of inhaled corticosteroids in COPD by record linkage analysis. Respiratory Research 2014 15:141. 